\title{
THE USE OF SANDPAPER IN THE PREPARATION OF HISTOLOGIC GROUND SECTIONS OF HARD SUBSTANCES
}

\author{
J. I. FANZ
}

Laboratories of the Daniel Baugh Institute of Anatomy of the Jefferson Medical College, Philadelphia

In the microscopic study of specimens of compact bone, teeth, shells, hard roots, hard plant stems, etc., the technician is often confronted with the very tedious and time-consuming process of grinding and honing in accordance with present methods.

The technique herein described was devised by the author to prepare thin microscopic sections of a certain very dry, hard and brittle plant stem. Sections ground and honed by present methods required hours for their production and ultimately contained little or no cork and were in most cases too thick and irregular for photomicrography. Clogging of the surface of the water hone, burning on the lathe, warping of the section by alternate wetting and drying, general faulting and fracturing of sections, are obstacles very discouraging to the beginner and require the skill of the initiated to be overcome.

By the introduction of sandpaper in ground-section technique, the time required to obtain thin, complete, workable sections was reduced to about one-fourth. The technique, of course, is not limited to botanic research, but is applicable to all lines of histologic work in which hard substances are to be studied.

\section{THE TECHNIQUE}

A characteristic portion of the specimen is exposed by a saw cut made with a jeweler's saw. The cross, longitudinal, tangential, or oblique cut is previously determined in accordance with the contemplated study. The exposed surface is then examined by means of a magnifier to ascertain if the required detail is 
present. The object is clamped in an ordinary carpenter's wood clamp or a small vise and a section 1 to $3 \mathrm{~mm}$. thick removed by saw cut. Great care is taken in sawing off this coarse section, the blade of the jeweler's saw always being kept parallel to the exposed surface of the section. Manipulation of the saw blade must be steady and light. With a little practice very thin coarse sections can be obtained, their relative thickness, of course, lessening the amount of subsequent grinding. Specimens presenting exceptional difficulties to sawing may sometimes be chipped or fractured and then small thin bits selected for grinding.

The next step is that of cementing the coarse section to a microscope slide preparatory to grinding. After numerous substances were tried, shellac was found best adapted for this purpose. Rather thick heavy slides, 1 inch $\mathrm{x} 3$ inches, were selected, washed in alcohol to remove possible grease, and dried. The coarse section is cemented near one end so that the slide can be firmly grasped and the index-finger pressed over the back of the section while grinding, insuring uniform distribution of pressure over the section. A pinch of dry scale orange shellac is placed on an old spoon (tea) and carefully heated just to and not beyond its melting-point. The melted shellac is then carefully poured around the coarse section, which must be held firmly against the glass to prevent the shellac from flowing under the section and raising it, thereby causing irregularities in grinding. Hot shellac sets very rapidly and the preparation is ready for grinding on the sandpaper in five minutes.

Grinding is accomplished entirely on sandpaper of different grades of coarseness, and the section is never dipped in water or oil as in present methods. Flint, sand, emery, or carborundum papers are selected according to the hardness of the material to be ground. The sand (flint) paper used was of following grades, Nos. 2, 1, 00, 0000. Sheets, $10 \times 12$ inches, were cut into pieces $3 \times 5$ inches and were held over a smooth, hard wood block, $3 \times 4$ inches, while grinding. Brittle substances grind best on No. 00, using a light touch. Tougher substances can be ground safely and more rapidly on Nos. 2 and 1 . The index- 
finger is placed on outer part of slide (outer third) directly opposite to the specimen, so as to insure uniform pressure to all parts of the coarse section, and the slide is moved with a quick, uniform, easy swing in an elliptical direction over the sandpaper surface. Particles must frequently be jolted off the surface to prevent scratching furrows. As soon as a paper becomes clogged or worn out in its entirety, it is discarded for a fresh piece. Old sheets are used in grinding prior to polishing, and some specimens even polish readily in their own dust on used papers. As soon as the first surface is ground true, it must be finished on No. 0000. Two pieces of this grade are rubbed faces together to remove large particles (to prevent scratching), and after this final grinding on No. 0000 , the section is ready for polishing. The ground surface is polished by rubbing on the back (smooth side) of a piece of sandpaper, the paper pulp constituting the buffing surface. Care must be taken to have no flint particles on this surface. Final polishing may be performed on a piece of smooth ground glass, on the palm of the hand, or on a razor strop.

After grinding and polishing one surface, the section must be removed, inverted, and recemented prior to grinding and polishing its second surface. To remove the section it is best to chip away the shellac zone and then soak the specimen in 95 per cent alcohol or methyl alcohol to soften the remaining shellac. The section may then be removed by slipping a razor blade under it and lightly lifting it off to another clean, dry slide. The polished surface is placed in contact with the second slide and the section is cemented on the outer third of slide with hot shellac in the manner given above.

In cutting down the second surface, care must be taken to grind it down in a plane parallel to the surface already completed. The precaution of preventing hot shellac from flowing beneath the specimen must again be borne in mind. Simply pour the shellac around the section's periphery, extending a shellac zone $\frac{1}{2}$ inch in width around the section to insure uniformity in grinding, as this really is a guide to prevent tapering at the edge of the specimen. Frequent measurements with the micrometer or examinations with the $\frac{1}{4}$-inch objective are necessary at the 
various stages of grinding. As the section loses its thickness, care should be increased and grinding pressure decreased. Towards completion the greatest care is necessary to prevent fracture, and it is well to examine section frequently with high-power objectives. Ultimate polishing of the second surface is performed in the manner described above, but greater care is necessary.

Removal of the thin polished section from the glass side is sometimes difficult. Chip away the excess shellac zone and submerge the slide horizontally in a Petri dish of 95 per cent alcohol. Very thin sections cannot be safely manipulated with the razor blade and sometimes require four or five hours' soaking in alcohol prior to their removal. Alcoholic stains may then be used directly, or dealcoholization followed by aqueous stains may be employed. Dehydration should be slow and the clearing and mounting in balsam with cover-glass should be performed as in the ordinary methods of technique. 